Meta

Journal des traducteurs

Translators' Journal

\title{
L'enseignement des langues dans le secondaire et la traduction
}

\section{Armand Roth}

Volume 28, numéro 3, septembre 1983

URI : https://id.erudit.org/iderudit/003995ar

DOI : https://doi.org/10.7202/003995ar

Aller au sommaire du numéro

Éditeur(s)

Les Presses de l'Université de Montréal

ISSN

0026-0452 (imprimé)

1492-1421 (numérique)

Découvrir la revue

Citer cette note

Roth, A. (1983). L'enseignement des langues dans le secondaire et la traduction. Meta, 28(3), 310-315. https://doi.org/10.7202/003995ar d'utilisation que vous pouvez consulter en ligne.

https://apropos.erudit.org/fr/usagers/politique-dutilisation/ 
L'enseignement des langues dans le secondaire et la traduction

Il sera question ici de la traduction comme auxiliaire de la pédagogie des langues dans le secondaire, et comme préparation éventuelle à une spécialisation dans le domaine de la traduction propre et de l'interprétation au niveau universitaire.

La seule mention du mot traduction fera frémir de nombreux professeurs de langues de l'enseignement secondaire, comme certains de l'enseignement supérieur, d'ailleurs. Depuis une quinzaine ou vingtaine d'années le mot est banni de leur vocabulaire, et la chose de leur pédagogie. Pour quelques-uns même le problème n'existe plus : il serait depuis longtemps enterré. Or l'ironie ou le paradoxe est que ces ensei- 
gnants, dans leur majorité, n'ont guère eu l'occasion de pratiquer la moindre traduction dans leurs classes ou cours, et, par conséquent, d'en vérifier la prétendue inefficacité pour l'acquisition d'une langue étrangère. Dans ce domaine ils se fient à ce que leur ont dit quelques aînés et des enquêtes réputées «scientifiques", et pour le reste ils font confiance aux méthodes nouvelles ${ }^{1}$ dont on sait que pour l'essentiel elles reposent sur la théorie qu'une langue étrangère devrait s'apprendre comme la langue maternelle, sans avoir recours au genre d'explication que représente finalement l'acte traducteur.

Attitude compréhensible quand on songe à ce qui se raconte généralement concernant le rôle de la traduction dans les méthodes en honneur dans un passé encore récent. Or l'honnêteté oblige à reconnaître que ce rôle a trop souvent été exagéré ou caricaturé, et qu'il a certainement varié d'un pays ou d'un système pédagogique à l'autre ${ }^{2}$.

Il est possible qu'au lendemain de la dernière guerre mondiale, dans les écoles secondaires de certains pays, l'enseignement des langues se soit encore souvent trouvé calqué sur celui du latin et ait pu se résumer quelquefois par la célèbre formule : «Lisez! Traduisez!» Dans d'autres écoles, cependant, la formule s'entendait plus souvent, et encore dans la langue étrangère, à la fin d'une leçon de quelque cinquante à cinquante-cinq minutes dont elle ne prenait que cinq ou dix au grand maximum. Il s'agissait en l'occurrence pour le professeur de vérifier si le texte (description, narration, dialogue, etc., d'une douzaine de lignes) qui avait été présenté selon une méthodologie défendable encore aujourd'hui : explication du vocabulaire et des structures regroupés autour de centres d'intérêt avec renforcement immédiat sous forme de questions, lecture du texte par le professeur, livre fermé chez les élèves, élucidation du texte sous forme d'un dialogue intensif, lecture par les élèves, présentation des faits grammaticaux (dans la langue maternelle cette fois-ci), traduction enfin, avait été effectivement compris.

Loin donc de représenter l'essentiel de la méthode, la traduction orale dans la langue maternelle n'intervenait qu'à titre de vérification ${ }^{3}$, "as a checking method", comme on dirait en anglais. L'expérience de nombreux professeurs de langues des plus chevronnés montre, en effet,

1. Elles-mêmes déjà plus si nouvelles puisque de nombreuses méthodes récentes accusent un certain retour au passé, $c e$ qui semble montrer que la pédagogie des langues, comme la confection féminine et masculine, connait elle aussi ses modes.

2. Jean Darbelnet (1963): «Pour une revalorisation des exercices de traduction dans l'étude des langues", Culture XXIV, p. 352

3. Vinay-Darbelnet (1971): Stylistique comparée du français et de l'anglais, Montréal, Beauchemin, p. 24. qu'un élève, pour avoir fourni des exemples dans de nombreux contextes nouveaux, peut donner l'impression d'avoir assimilé le mot, la structure, le tour, l'expression idiomatique que le professeur vient d'expliquer et d'illustrer. Cependant, bien souvent, seule la traduction dans la langue maternelle prouvera de façon concluante que le sens de ces éléments a été effectivement saisi.

Mais là n'est pas, bien entendu, l'essentiel de notre propos. Il s'agissait tout d'abord de déblayer quelque peu le terrain et de montrer, avant de pouvoir discuter objectivement du rôle possible de la traduction dans l'étude des langues, que l'importance et l'objet de celle-là ont été trop souvent dénaturés à tel point que de nombreux professeurs de langues n'acceptent même plus d'en discuter.

Pourtant, l'expérience de la vie en général, et de la pédagogie en particulier, devrait nous inciter à un peu de modestie. Dans le domaine des langues comme dans d'autres disciplines, l'acquis du passé n'est jamais entièrement à rejeter. Or, depuis une trentaine d'années, que de méthodes et approches soi-disant radicalement nouvelles dans la pédagogie des langues vivantes, et qui toutes ont connu des sorts variables. Le malheur est que trop souvent elles ont été présentées comme des solutions uniques et qu'elles ont trouvé des défenseurs enthousiastes qui finissaient par ne jurer que par elles. Ces défenseurs invoquent comme témoin de l'efficacité des méthodes nouvelles le fait qu'une plus grande proportion de jeunes gens possède maintenant une deuxième langue. Encore convient-il de s'interroger: $I^{\circ}$ si les chiffres qu'ils avancent à l'appui de leur thèse sont des chiffres relatifs ou absolus. En d'autres termes la proportion d'élèves qui arrivent à une bonne mâ̂trise de la langue étrangère est-elle tellement plus élevée qu'il $\mathrm{y}$ a vingt ou quarante ans. $2^{\circ}$ dans 1 'affirmative, si cette maîtrise ne tient pas plutôt au fait que les occasions de voyages et de séjours à l'étranger sont actuellement plus nombreuses qu'avant la guerre. Ce sont là des questions qui méritent d'être soulevées. L'impression de progrès dans la pédagogie des langues est souvent attribuable au fait que la minorité «douée» pour les langues et dont on peut dire qu'elle apprend souvent «en dépit» du professeur et de la méthode ou qui a appris "sur le tas», est actuellement beaucoup plus importante pour les raisons que l'on devine aisément : importance et prestige de certaines langues, débouchés plus nombreux offerts aux bilingues, comme au Canada, par exemple, etc.

Restent donc ces élèves du secondaire et ces étudiants du supérieur, les plus nombreux, pour qui le problème de l'efficacité de nos méthodes, que ce soient celles du passé ou du présent, semble rester entier. Il est légitime dans 
leur cas de se demander si les exercices traditionnels de la traduction appelés version et thème peuvent trouver une place dans l'enseignement qui leur est destiné.

On a vu ci-dessus que la version pratiquée à la fin de la leçon comme simple méthode de vérification présentait l'avantage pour le professeur de pouvoir s'assurer que le vocabulaire, les expressions idiomatiques, les structures de la langue étrangère ont été bien expliquées, et pour l'élève de montrer qu'il les a bien compris. L'exercice est donc profitable aux deux, à condition $I^{\circ}$ que le texte dans la langue de départ soit court (une dizaine ou douzaine de lignes), qu'il présente une certaine unité de ton et de niveau de langue et soit rédigé dans la langue de notre époque; $2^{\circ}$ que les élèves ne soient pas trop jeunes, c'est-àdire, qu'ils aient au préalable acquis les structures et le vocabulaire de base.

En effet, un texte trop long n'en permettrait pas une traduction correcte et idiomatique en quelque dix minutes; et un âge trop jeune ne garantirait pas chez l'élève une connaissance suffisante de sa propre langue, surtout au niveau du vocabulaire abstrait et des tours de langue. Cette remarque est d'autant plus valable à une époque où sous l'influence de l'audio-visuel l'écart entre l'aptitude à l'expression orale et à l'expression écrite semble se creuser toujours davantage.

Ces quelques indications devraient montrer encore une fois qu'il ne s'agit pas, en l'occurrence, de revenir à la version comme méthodes d'acquisition qui occuperait l'essentiel de la leçon, mais bien comme méthode de vérification en temps limité.

L'autre avantage que présente la version ainsi pratiquée à la suite d'un texte expliqué, est que l'élève est amené, dès qu'il possède l'indispensable maîtrise des mécanismes de base, à comparer les deux langues en présence. Cette comparaison lui apprendra très tôt que ces langues, la langue étrangère qu'il est en train d'étudier, sa langue maternelle dont il est loin de posséder toutes les subtilités, appréhendent la même réalité de façon différente. Les deux langues, pour emprunter une image au domaine des moyens de communication, sont comme deux trains menant au même endroit, mais roulant souvent à des vitesses différentes, sur des voies différentes et qui sont loin d'être toujours parallèles. Après des années d'étude de la langue de nombreux étudiants anglophones restent toujours aussi mystifiés devant les équivalents anglais-français: I miss her - elle me manque. Amener l'élève à réfléchir sur cet exemple qu'on pourrait multiplier indéfiniment devrait développer en lui une attitude plus ouverte et plus circonspecte aussi à l'endroit des phénomènes linguistiques qui varient d'une lan- gue à l'autre. Il est difficile de dire à partir de quel moment la traduction ou comparaison de deux façons de penser amène l'élève, au-delà des différences linguistiques, à prendre concience des différences culturelles que les premières véhiculent. Au niveau qui nous intéresse, la chose n'est pas essentielle, mais si elle a lieu c'est autant de gagné. Ce que Jean Darbelnet a dit à ce sujet, il y a une vingtaine d'années déjà, est particulièrement intéressant à lire ou à relire ${ }^{4}$.

Un dernier avantage de la version nous semble-t-il, et certains seront étonnés de le voir mentionner ici, est qu'elle nous aide à mieux soigner notre propre langue : notre langue maternelle ou notre deuxième langue si nous sommes des immigrés relativement récents. On objectera que l'essentiel dans la pédagogie des langues est finalement d'apprendre la langue étrangère. À quoi l'on répondra que la personne qui ne s'embarrasse ni de correction, ni de précision dans sa propre langue aura rarement davantage le souci de la correction grammaticale, celui de la nuance, du mot juste, dans l'autre langue. Nous oublions trop facilement que dans le domaine linguistique comme dans tant d'autres, tout se tient. Vouloir compartimenter les choses serait une erreur grossière. Le professeur d'anglais ayant uniquement affaire à des francophones, ou le professeur de français enseignant surtout à des anglophones, aurait, s'il pratiquait la version, les plus grands torts de se contenter chez ses élèves, d'approximations dans le domaine de la grammaire, du vocabulaire, des expressions idiomatiques et tours de langage. Comment pourrait-il seulement employer la version comme méthode de vérification, et surtout de comparaison? Au fond, ce dernier avantage que représente pour la langue maternelle une traduction précise et idiomatique est loin d'être superfétatoire, il découle logiquement et comme par surcroît des deux précédents. Bien s'exprimer dans sa propre langue entraîne à bien s'exprimer dans l'autre.

Un cas particulier est celui des immigrés qui, dans un pays comme le Canada, aimeraient posséder les deux langues officielles. La pratique de la version à partir de la deuxième langue dans la première présenterait certainement beaucoup d'intérêt ${ }^{5}$.

Ayant rapidement passé en revue quelquesuns des avantages de la pratique de la version dans les conditions précisées, qu'en est-il du thème?

L'exercice de traduction dans la langue seconde ou la langue étrangère qu'on est en train

4. Jean Darbelnet, article cité, p. 353.

5. Le problème se complique évidemment du fait que les élèves immigrés de la deuxième génération se situent gépéralement, et encore plus que leurs camarades nés dans le pays, à des niveaux de compétence linguistique dans le pays, à des niveaux de compétence
différents dans la première langue de leur choix. 
d'apprendre, et qui est probablement le plus profitable dans la mesure où il constitue un renforcement du vocabulaire et des structures vus au cours de la présentation du texte, est ce qu'on appelle traditionnellement le thème d'imitation.

Il est important que celui-ci ne mette en cuvre que l'essentiel du vocabulaire, des structures et expression du texte original. La règle qui veut qu'en pédagogie des langues il est préférable de faire peu mais bien s'impose encore davantage ici que dans tout autre exercice. $\mathrm{Si}$ le cadre de la leçon traditionnelle de 50 minutes ou moins, suivant le système d'enseignement en vigueur, ne permet pas la pratique immédiate du thème d'imitation, celui-ci devrait faire l'objet d'une préparation à la maison et d'une correction lors de la première leçon suivante. Le thème d'imitation présente l'avantage de reproduire autant que possible des situations qui s'intègrent dans un cadre assimilable à ceux de la vie réelle. Bien choisi il accusera de nombreuses similitudes avec le texte étudié dans la langue étrangère dont il reproduira autant d'éléments importants que possible. On sait que les descriptions, narrations, dialogues d'auteurs qui écrivent simplement sont le plus souvent supérieurs à ceux fabriqués par des auteurs de manuels et de méthodes reproduisant des situations factices et des dialogues artificiels.

En dehors des éléments purement linguistiques qu'il livre à la compréhension et à la pratique des élèves, le thème d'imitation présente encore l'avantage de renforcer la connaissance des éléments culturels que véhicule toute langue authentique. En effet, s'il est bien choisi, le texte de départ n'est pas quelque chose d'abstrait, mais est représentatif d'un peuple d'un endroit et d'un moment donnés. I comporte un contexte qui en précise et nuance les éléments culturels.

La même chose ne vaut pas pour la traduction en langue étrangère de phrases isolées. Cependant, ce genre d'exercice est loin d'être inutile à condition de ne prêter à aucune ambiguité grammaticale ou sémantique. La traduction de phrases indépendantes, hors contexte, se pratique généralement dans le cadre du renforcement de règles grammaticales plutôt que du vocabulaire et de tours subtils. C'est d'ailleurs l'exercice idéal pour ceux qui ne croient pas à l'enseignement traditionnel ou théorique de la grammaire, et pour qui c'est une occasion de remonter de la pratique, des exemples, à la théorie.

Aucune méthode d'enseignement des langues, à notre connaissance, n'a encore résolu le problème épineux de l'existence des règles grammaticales que d'une façon ou d'une autre l'élève doit appliquer. Prenons l'exemple bien connu des professeurs de français s'adressant à des anglophones, ou vice versa, et qui concerne l'expression d'une action commencée dans le passé et qui se prolonge dans le présent. A moins de placer quelqu'un pendant une période prolongée dans un milieu francophone où il entendra à longueur de journée le présent là où en anglais on emploie le «present perfect», on peut honnêtement se demander quelle méthode, structuraliste ou autre, viendra à bout de la difficulté de l'emploi d'un temps différent dans les deux langues, si elle ne se livre pas à un certain moment et dans ce domaine à une étude comparative des mécanismes des deux langues en présence. Les exercices de substitutions répétées, pratiqués au laboratoire et à un moindre degré dans la salle de classe, et qui visent à développer un réflexe qui ferait que l'élève, en entendant: depuis, il y a, cela fait, voilà... emploirait automatiquement le présent ou l'imparfait suivant les circonstances, ne sont pas toujours suivis d'effets durables. La traduction écrite ou orale de phrases isolées amène l'élève, de façon répétée, à prendre conscience de la différence avec laquelle l'anglais et le français appréhendent la même réalité temporelle. Cette prise de conscience, plutôt qu'une répétition mécanique, a de meilleures chances d'entraîner des résultats chez une majorité de sujets. Ce qu'on oublie trop souvent, c'est que l'élève, qu'on le veuille ou non, traduit malgré lui et surtout malgré le professeur. Donc, à la différence du thème d'imitation dont le but est de renforcer l'assimilation du vocabulaire, des structures, des tours de phrase, des expressions idiomatiques dans la langue écrite et dans une situation donnée dont les éléments s'éclairent mutuellement, la traduction de phrases isolées, précises et hors contexte vise le plus souvent à l'élucidation d'un problème de grammaire précis comportant une solution unique ou, tout au plus, un nombre limité de variantes. Prenons: « He had been sleeping for half an hour when the phone rang», phrase qui présente deux sortes de problèmes pour l'élève francophone: $1^{\circ}$ celui de la traduction d'un concept de temps différent : le pluperfect anglais devant être traduit par l'imparfait français; $2^{\circ}$ celui de savoir s'il s'agit de la langue parlée ou de la langue écrite soutenue, littéraire, ce qui obligera l'élève dans un cas à employer le passé composé, dans l'autre le passé simple ou défini. Seule la traduction écrite ou orale qui accorde à l'étudiant le temps pour bien formuler sa phrase, qui autorise l'hésitation et l'autocorrection lui donnera l'occasion de pratiquer une certaine stylistique comparée. Et plus il avancera en âge, plus ce réflexe entraînant la comparaison entre sa langue maternelle et la langue étudiée sera vif. Passe que l'on demande à de jeunes élèves, dont l'enthousiasme, la spontanéité et les simples facultés mimétiques sont particulièrement vives, de répéter, quelque- 
fois sans comprendre, des structures de la langue étrangère qu'ils commencent à étudier; il est difficile de prolonger cette "approche" audelà d'un certain âge et nombre d'années d'études, et surtout de l'essayer sur des adultes. Le résultat est presque toujours une frustration réciproque aboutissant en fin de compte à l'exaspération d'un côté et à l'ennui de l'autre.

Quelques phrases de thème détachées de tout contexte, mais simples et précises peuvent donc présenter, à la fin de la leçon, une excellente base pour l'élucidation des difficultés grammaticales, que ce soit à l'occasion d'un premier contact avec la règle ou d'une révision.

Il est évidemment possible de trouver un moyen terme entre les deux genres d'exercices: thème d'imitation, phrases détachées de tout contexte. De nombreux manuels, dont certains étaient depuis longtemps en usage, proposaient en effet aux élèves la traduction de phrases mettant en cuvre à la fois les règles de grammaire et le vocabulaire et les expressions idio. matiques illustrés dans le texte formant la base de la leçon.

Jusqu'ici on a surtout évoqué les vertus d'un certain type de version et de thème dans le cadre de la pédagogie des langues; mais ainsi pratiqués, ces exercices présentent encore l'avantage de pouvoir susciter des vocations d'interprète et de traducteur, d'initier de futurs étudiants en traduction et interprétation à un art qu'ils pratiqueront en amateurs ou professionnels.

On imagine tout de suite l'objection de nombreux professeurs de langues qui estimeront que leur tâche n'est pas d'enseigner la traduction mais la langue. A quoi l'on répondra que si le professeur de langues peut faire de la traduction dans des limites strictement définies et comme partie intégrante de la pédagogie des langues et inculquer à la même occasion quelques notions de base pouvant servir à ceux qui manifesteront un peu plus tard du goût pour la traduction, il y a de bonne chance pour que ce professeur fasse d'une pierre deux coups.

On sait, bien sûr, qu'il ne suffit pas d'être bilingue pour s'improviser traducteur et, une fois de plus, il n'est pas question de demander aux professeurs du secondaire d'enseigner "l'art» de la traduction. Mais que vaudrait un professeur de langues qui ne saurait pas élaborer correctement un nombre restreint d'exercices de traduction à partir de sa propre langue qui est aussi le plus souvent celle de ses élèves, et qui serait incapable, par exemple, de faire en sorte que ceux-ci sachent traduire de façon idiomatique : "She swam across the river», par: elle a traversé la rivière à la nage? On peut s'interroger sérieusement sur la compétence d'un tel professeur à ce niveau.
Et l'on est surpris de voir quelquefois dans les écoles de traduction et d'interprétation des étudiants qui n'ont jamais fait de traduction dans le secondaire. Examens et tests d'admission dans les écoles de traduction dont les programmes font immédiatement suite à ceux du secondaire vérifient surtout certaines aptitudes linguistiques dans les deux langues (la langue maternelle et la langue étrangère ou langue seconde) dans lesquelles les candidats se proposent de travailler. L'exception est constituée par ceux des étudiants qui s'inscrivent à une mấtrise en traduction ou à un diplôme institué au niveau des études supérieures (graduate studies) sans que ces études se placent nécessairement et administrativement parlant dans le cadre de la Faculté des Études Supérieures (Faculty of Graduate Studies). Il est probable dans ce dernier cas que l'étudiant aura été initié à la traduction au cours des trois ou quatre années menant au baccalauréat (B.A.) ou à la licence. La plupart des programmes des départements de langues des universités canadiennes, et certainement des universités européennes, comportent au moins un ou deux cours de traduction à titre obligatoire ou facultatifs ou à option.

Il n'est pas sûr que les professeurs du secondaire donneraient nécessairement de mauvaises habitudes à leurs élèves si leur propre formation dans les facultés d'éducation ou centres pédagogiques comportait, comme c'est le cas dans de nombreux pays, une initiation sérieuse ou un entraînement systématique à la pratique de la version et du thème, à condition que cette pratique se fasse dans une langue correcte, idiomatique et accessible à la majorité. Pas de traduction littéraire ou technique, par conséquent, mais une traduction de la langue de tous les jours. Ceci nous obligerait sans doute à revoir certaines de nos attitudes vis-à-vis de la traduction : celle de ceux qu'on pourrait appeler les « littéraires» parmi les professeurs de langues, qui veulent faire aborder rapidement, trop rapidement, l'étude des textes à valeur littéraire et culturelle, mais dont la langue est trop souvent archaïque et abstraite; celle de ceux qu'on pourrait appeler les «linguistes purs», pour qui le moindre exercice de traduction, quelle que soit d'ailleurs la nature du texte employé, est une trahison ${ }^{6}$. On ne soulignera jamais assez à l'endroit de ces derniers qu'il ne s'agit nullement de revenir aux habitudes, extrêmes dans certains cas, qui consistaient à faire de la traduction la base même de la pédagogie des langues, mais de voir en quoi la version et le thème, pratiqués sous les formes indiquées ci-dessus, dans des conditions rigoureuses et avec des

6. Trahison de leur méthode. (Il ne s'agit pas, bien entendu, de cette minorité infime qui estime que l'acte traducteur de cette minorite infime qui estime que l'acte traducteur
est un acte impossible et pour qui, dans un sens absolu, traduction $=$ trahison) 
objectifs limités, pourraient être intégrés dans un enseignement revitalisé.

Pour conclure, la traduction peut servir à la fois d'auxiliaire précis pour tous ceux, la majorité sans doute, dont le souci principal est d'apprendre la langue étrangère ou seconde, et pour ceux aussi qui resteront toujours une minorité et dont le but est d'apprendre la langue et de se préparer insensiblement à une profession dont l'attrait et l'importance sur le marché du travail sont de plus en plus évidents.

ARMAND RoTH 